\title{
A Study on the Relationship Between Childhood Abuse or Neglect and Adult Violence
}

\author{
Jiayu Huang ${ }^{1, *}$ \\ ${ }^{1}$ psychology, Oregon State University, Corvallis, America \\ *Corresponding author. huangj6@oregonstate.edu
}

\begin{abstract}
In a longitudinal study in Sweden, whether people who have been abused or neglected by their parents in childhood are more prone to violence and mental illness in adulthood. Therefore this paper focuses on the relationship between childhood abuse or neglect and adult violence.

Subjects were male $(\mathrm{n}=199)$, recruited from high-risk communities and grouped according to childhood abuse and neglect index variables, resulting in high $(\mathrm{n}=110)$ and low victimization $(\mathrm{n}=89)$ groups. In order to facilitate comparison and resolution, a comparison table is set for comparison, and the method of configuration frequency analysis (CFA) is used to determine its contingency.

Through the comparison of experimental data, it can be seen that children who are severely abused or neglected in childhood are more likely to produce extensive violence and higher PCL-R test scores in adulthood, and higher PCL-R scores will also lead to more extensive violence. Those who were abused or neglected in childhood, who were mild or none at all, showed mild \{or no\} violence. Thus, it can be concluded that there is a close relationship between PCL-R, the degree of childhood abuse or neglect and violence.
\end{abstract}

Keywords: Abuse, neglect, children, violence, mental disease

\section{INTRODUCTION}

Violence is considered the most common crime. Over the years, many studies have shown that adult violence is caused by different types of abuse or neglect by caregivers in childhood. Federal legislation provides guidance to the states by establishing minimum acts or practices that define child abuse and neglect. The federal child abuse prevention and Treatment Act (CAPTA), as amended by the CAPTA reauthorization act 2010, defines child abuse and neglect as at least:"Any recent act or omission of a parent or caregiver resulting in death, serious physical or emotional injury, sexual abuse or exploitation";[1] perhaps"An imminent risk of serious injury from an act or omission."According to Bandura's theory and many studies, violent and psychiatric patients often come from families where parents are aggressive and do not pay attention to their children's needs. Subsequently, Cathy Spatz widom in its own experiment compared the official criminal history of a large number of confirmed and verified cases of physical and sexual abuse and neglect from 1967 to $1971(\mathrm{n}=908)$ with the matched control group $(\mathrm{n}=667)[2]$. Abused and neglected subjects had a higher rate of adult criminal records than the control group, and were arrested more often in adulthood. It is further concluded that early childhood abuse or neglect is not only easy to lead to violence, but also may escalate into crime. But at the same time, there is a reality that mental patients are more likely to commit violence than normal people. According to dsm-5, one way to diagnose antisocial personality is through violence: "very low tolerance to friction and a low threshold for discharge of aggregation, including violence". According to hare, although psychiatric patients account for only about $1 \%$ of the total population, they account for a large proportion of violence and social difficulties in every society. They account for $15 \%-20 \%$ of prison inmates in the United States and are common among those who abuse their spouses and children, gang members and terrorists. At the same time, PCL-R has proved to be a very effective method to predict crime[3]. So what is the relationship between violence and psychosis and early childhood abuse? That's what this article wants to discuss. This paper studies the relationship between childhood abuse and growing up violence and psychosis. 


\section{HYPOTHESIS}

According to the above research and theory, this study puts forward the following assumptions for the experiment:

First Subjects who reported neglect or abuse in childhood were more likely to get a higher PCL-R test score in adulthood. Second subjects with higher PCL-R scores are prone to more extensive violence. Third Being abused and neglected in childhood is not the direct cause of violence, and mental illness may play a mediating role in childhood.

\section{METHOD}

\section{1: PCL-R TEST}

Hare PCL aims to assess the presence and extent of psychosis in a standardized and reliable manner. It measures two stability factors; On the one hand, it refers to a series of interpersonal and emotional characteristics, such as self-centered and lack of regret. On the other hand, it refers to the psychotic aspects of impulse, antisocial and unstable lifestyle. The PCL rating was conducted in a follow-up interview with adult subjects. A psychologist rated each topic based on information from about three hours of in-depth recorded interviews. A simplified version of the PCL, including 13 items (Table 1), was used and did not rate whether the subjects belonged to the original crime group or the control group[3].

Table 1. PCL-R test form

\begin{tabular}{|c|c|}
\hline PCL1. & Glibness, superficial charm \\
\hline PCL2. & Grandiose sense of self-worth \\
\hline PCL3. & Need for stimulation, proneness to boredom \\
\hline PCL4. & Conning, manipulative \\
\hline PCL5. & Lack of remorse or guilt \\
\hline PCL6. & Shallow affect \\
\hline PCL7. & Callous, lack of empathy \\
\hline PCL8. & Poor behavioral controls, prone to lose temper (even for trifling matters) \\
\hline PCL9. & Lack of realistic, long-term plans \\
\hline PCL10. & Impulsivity, poor ability to foresee consequences of own actions \\
\hline PCL11. & Irresponsibility, does not complete commitments \\
\hline PCL12. & Many short-term marital relationships \\
\hline PCL13. & Failure to accept responsibility for own actions \\
\hline
\end{tabular}

\subsection{Measurement of criminal violence}

The crime data of subjects aged 11-14 were from the original study, while the crime data of subjects aged 15 and over were from the follow-up sociological study and the National Police Commission. Signs of violence range from 11 to 14 years of age and earlier, and up to adulthood ( 38 to 46 years of age). The following indicators of violence are summarized as indicators of violence. The number of violent acts is 1 , while those without violence are 0 .

\subsection{Definition of violence}

The definition of violence should fully explain the exclusion of acts such as accidents and self-defence, as well as acts including child abuse, sexual crimes and manslaughter. Violence research has produced many sometimes contradictory definitions of violence, which can be divided into four general camps: paradigm method, social psychology method, public health method and animal research method. Each approach has strengths and limitations, but to completely distinguish violence from other acts, all these elements need to be combined. The comprehensive definition of violence includes four basic elements: (a) intentional, (b) unwanted, (c) unnecessary and (d) harmful behavior. Some elements require more complex identification.[4]

\section{RESULT AND ANALYSIS}

In terms of the degree of abuse and neglect of childhood, six characteristics are adopted as the dividing point. That is, if six or more conditions of abuse and neglect are met, they will be regarded as high-level victims, otherwise they will be regarded as low-level victims. Victims with a high degree of victimization will be identified as number 1 . Therefore, among the 199 subjects, 110 had low or no experience of abuse and neglect, and 99 had a high degree of abuse. At the same time, the dichotomy is adopted for whether it is 
widespread violence. Through the above definition of violence, if it meets more than four definitions of violence, then these subjects are considered to have widespread violence. There was no or mild violence $(\mathrm{n}=$ $152)$ and extensive violence groups $(n=47)$. Divided into PCL groups at three intervals. Distribution approximately $46 \%-27 \%-27 \%$ of subjects entered the low $(n=98)$, medium $(\mathrm{n}=52)$ and high $(\mathrm{n}=49)$ PCL groups.

Figure 2 depicts the relationship between childhood victims and violence. The significance is calculated. It can be seen that $90 \%$ of the group with low degree of childhood abuse or contempt showed a low tendency of violence; In the extensive violence test, the number of subjects with low degree of victimization is less than half of that accidentally expected. Among the high victimized subjects, those with low tendency of violence were also lower than expected.

Table 2:The frequency of SS obtained (and expected) was divided into low victimization $(\mathrm{n}=110)$ and high victimization in childhood $(n=89)$ groups according to the measurement of violence; No or slight indication ( $\tilde{\mathrm{N}}$ $=152)$, as well as extensive $(\tilde{\mathrm{N}}=47)$ beyond puberty and adulthood, and significance levels were assigned to each group

Table 2. The degree of neglect in childhood reports is related to the extent of violence

\begin{tabular}{|c|c|c|c|}
\hline & $\begin{array}{l}\text { Violence } \\
\text { None/minor }\end{array}$ & Extensive & $n$ \\
\hline Low victimization & $99(84.0), P=0.0193$ & $11(9.25), P=0.0004$ & 110 \\
\hline High victimization & $53(67.9), P=0.0139$ & $36(21.0), P=0.001$ & 89 \\
\hline$n$ & 152 & 47 & 199 \\
\hline
\end{tabular}

The data in Figure 3 analyzes the relationship between PCL-R score and widespread violence. Low PCL $(\mathrm{n}=98)$, medium PCL $(\mathrm{n}=52)$ and high PCL $(\mathrm{n}=$ $49)$ were measured by violence in adulthood; No or slight $(n=152)$ and extensive $(n=47)$ in adolescence and adulthood. The main purpose of Figure 3 is to find out whether there is a certain connection between PCL-R and widespread violence through comparison. According to the PCL-R test, we get mental diseases. First, we divide violence into extensive or mild and none. Secondly, it is divided into low, medium and high according to the score of subjects' pcl-r. Secondly, according to the data distribution, the final conclusion is drawn. It can be seen that there were 98 subjects with low PCL-R score, while only 6 showed extensive violence, and the remaining 92 showed mild or no violence. Among the subjects with high PCL-R, the number of extensive violence was higher than that without or slight violence. And only high PCL-R test scores, the number of people in the group with widespread violence was higher than that with mild or no violence. It can be concluded that high PCL-R [i.e. psychosis] is closely related to widespread violence.

Table 3: The testers were divided into low PCL $(n=98)$, medium PCL $(\mathrm{n}=52)$ and high PCL $(\mathrm{n}=49)$, which were measured by violence in adulthood; No or slight $(n=152)$ and extensive $(n=47)$ in adolescence and adulthood

Table 3. The score of PCL-R test is related to the extent of violence

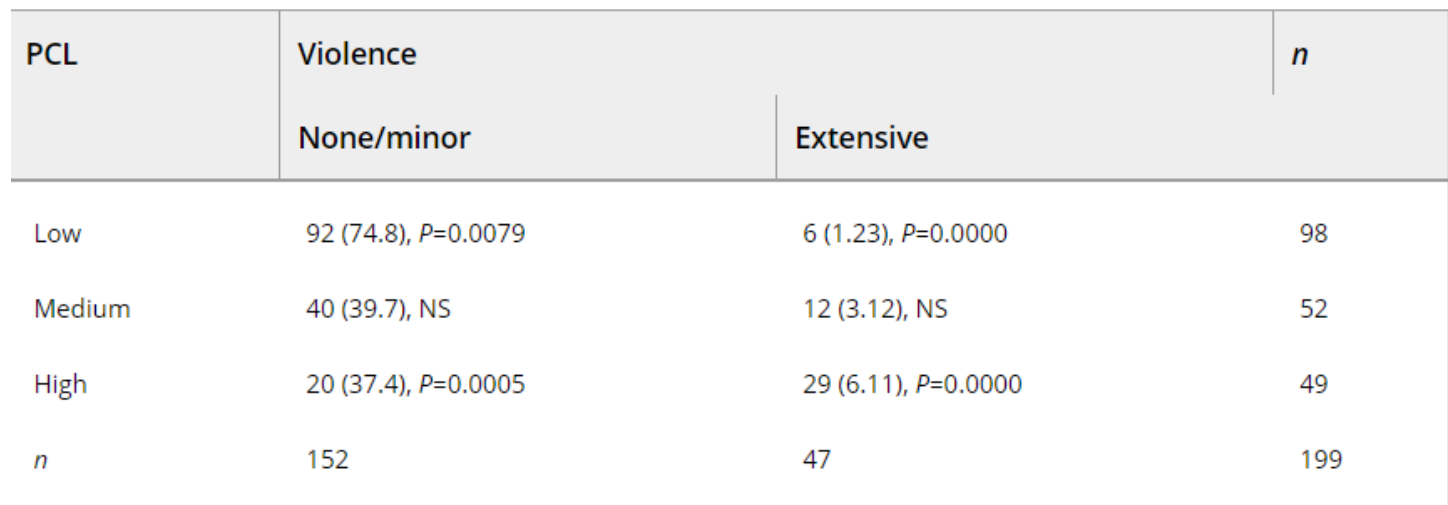

The adjusted significance level was obtained by multiplying the nominal significance level by 12 (the number of all possible combinations of variables in this design).
The table in Figure 4 is a collection of data by grouping the PCL-R score, the degree of abuse and neglect in childhood and whether violence is widespread. In order to facilitate a clear comparison, we can first see that those with high scores in the PCL-R test will indeed lead to widespread violence. The hypothesis that more 
victimized children are predicted to exert more violence than less victimized children is confirmed by the distribution, which shows that the presence of widespread violence SS in more victimized subjects is significantly increased. Among the broad categories of violence, only 11 of the 110 low victimized SS were observed. The average violence score of the group with high victimization was also much higher than that of the subjects with low victimization. Given the socioeconomic environment of the subjects and their families, the results can be interpreted as indicating that the prevalence of violence in the current materials is slightly higher than that in the representative sample of the normal population.

Table 4. Dichotomous structure of child victimization (low / high)

\begin{tabular}{|c|c|c|c|c|c|c|c|}
\hline \multicolumn{3}{|l|}{ Pattern } & \multicolumn{2}{|l|}{ Size } & \multicolumn{3}{|c|}{ Level of sign } \\
\hline Victimization & Extensive violence & PCL scores & Obt & Exp & $x^{2}$ & Adj sign & Type-antitype \\
\hline Low & No & Low & 78 & 57.9 & 6.95 & 0.02 & $\mathrm{t}$ \\
\hline High & No & Low & 14 & 16.9 & 0.51 & & \\
\hline Low & Yes & Low & 4 & 17.9 & 10.80 & 0.001 & at \\
\hline High & Yes & Low & 2 & 5.2 & 1.99 & & \\
\hline Low & No & Medical & 29 & 30.7 & 0.01 & & \\
\hline High & No & Medical & 11 & 8.9 & 0.45 & & \\
\hline Low & Yes & Medical & 6 & 9.5 & 1.29 & & \\
\hline High & Yes & Medical & 6 & 2.7 & 3.73 & & \\
\hline Low & No & High & 19 & 28.9 & 3.43 & & \\
\hline High & No & High & 1 & 8.5 & 6.58 & 0.02 & at \\
\hline Low & Yes & High & 18 & 8.9 & 9.13 & 0.05 & $\mathrm{t}$ \\
\hline High & Yes & High & 11 & 2.6 & 26.85 & 0.001 & $\mathrm{t}$ \\
\hline
\end{tabular}

\section{DISCUSSION}

According to Bandura's principles, psychiatric patients with mental illness often come from families where parents are aggressive and do not pay attention to the needs of their children. One of the best predictors of psychiatric development in young people is that the father is a psychotic, alcoholic or antisocial father[8]. Parental delay or inconsistent discipline is considered to be the reason when to resist impulse and temptation and lack of guilt; If the response is stimulated or reinforced by fear, individuals with psychosis show weaker results than normal people when learning classical conditional response. However, this is not the only condition According to the comparative analysis in Table 4 , it is difficult to conclude that there is no significant correlation between mental illness and child abuse. According to the analysis of the experimental data in Figure 4, among the 49 people with high PCL-R scores, only 12 self-reported child abuse or neglect, indicating that there is no significant relationship between child abuse and mental illness, so hypothesis 1 is wrong.
According to the data in table 3, 29 of the 47 extensive violence testers obtained higher scores in the PCL-R test, which is much higher than those with medium or low PCL-R scores. Therefore, the second hypothesis predicted before the experiment: subjects with higher PCL-R scores are prone to more extensive violence is correct, High PCL-R test scores do lead to more widespread violence in adulthood. The PCL-R score represents psychosis, so it can be inferred that psychosis does lead to broader violence in adulthood, which is consistent with our expectations and common sense.

According to the experimental data in Figure 2 and figure 4 , it is still difficult to make hypothesis 3 . Because there is no direct evidence to prove that the PCL-R score is related to childhood abuse, it is difficult to determine whether there is a mediating relationship between mental illness and childhood abuse. However, according to the research of Weiler and widom, there is no significant correlation between childhood victimization and mental illness[5]. They found a significant relationship between childhood victimization and psychosis and between child 
victimization and violence, which was explained as mediated by psychosis. On the contrary, the current results suggest that the association between childhood victimization and adult psychosis may be mediated by psychosocial factors, indicating the formation of a common basis of violence in vulnerable groups. Therefore, children living in abusive families with psychiatric related characteristics and behaviors, parental impulse and impulse control disorders, ADHD, alcoholism, poor social communication and low tolerance for setbacks are easy to witness and develop violence.

Because the experiment adopts a longitudinal project in Sweden, and the subjects are 199 men randomly sampled from high-risk communities in Sweden, it is difficult to popularize. The representation of the experimental data is limited, so it should be adopted in a wider range and random sampling at all levels of society in the future. Such an experiment will be more representative. Secondly, the samples of this experiment are from mild child abuse samples, while Weiler and widom studies are more serious child abuse samples, and they study American child samples, in which there are more serious examples, such as sexual abuse and physical violence. However, many data in this experiment still support Weiler's research, indicating that some reasons are still potentially related, although the experimental data representation has limitations.

It should be noted that in these experimental data, it can be seen that abuse in childhood will affect violence in adulthood, but this does not mean that higher abuse and neglect in childhood will directly lead to criminal behavior. As the experimental data indicate, the subjects with high childhood abuse and neglect experience also have low or no violence. Therefore, the high degree of abuse or neglect in childhood is related to violent crime, but it can be changed through behavior correction or family environmental experience, so children's experience does not directly lead to violent crime. Childhood abuse and neglect cannot be directly associated with high PCL-R [i.e. mental illness]. Finally, PCL-R has indeed been shown to produce higher violence. Therefore, it may be necessary to emphasize that risk groups for developing psychosis, such as hyperactivity aggression, children with conduct disorders and antisocial personality, who experience more serious victimization, We believe that there is a genetic rather than a social ultimate cause of the disease. The types of social causes proposed (e.g., child sexual / physical abuse) should improve emotional responses rather than lead to a reduction in specific forms of responses seen in psychosis. According to R.J.R. Blair that genetic factors are the emotional dysfunction at the core of mental illness[6]. Although the effects of this contribution have not been understood at the molecular level, we believe that the damaged nervous system includes the amygdala and orbital / ventrolateral frontal cortex. At the cognitive level, the destruction in these two key systems is reflected in the impairment of the ability to form stimulus reinforcement Association and change stimulus response Association as a function of accidental change should carefully participate in appropriate and available intervention plans at a very young age.

\section{CONCLUSION}

This paper studies the relationship between childhood abuse and growing up violence and psychosis. According to this experiment, the following conclusions are drawn. First, the higher degree of abuse in childhood is indeed related to violence, and the higher PCL-R test score than widespread violence has also been confirmed. However, there was no significant correlation between the degree of child abuse and the score of PCL-R test. Another hypothesis that psychosis is a mediator between child abuse and widespread violence has not been proved.

\section{ACKNOWLEDGMENT}

The research in this study was realized by accessing the data of the longitudinal research project young lawbreakers as adults (yla). The planning, implementation and financing of data collection of yla project are the responsibility of Professor s. ahnsj ö, Professor g. Carlsson and associate professor K. humble. Special thanks to r. Ö sterman for his excellent maintenance of yla database.

\section{REFERENCES}

[1] REBECCA M. BOLEN. Guardian Support of Sexually Abused Children: A Definition in Search of a Construct. SAGE Journals. Retrieved from. (January 1,2002) https://doi.org/10.1177/15248380020031003

[2] Barbara Luntz Weiler,Cathy Spatz Widom PhD. Psychopathy and violent behaviour in abused and neglected young adults. CBMH. Retrieved from (September https://onlinelibrary.wiley.com/doi/abs/10.1002/cb m.99

[3] Hare RD, Hart SD, Harpur TJ. Psychopathy and the DSM-IV criteria for antisocial personality disorder. J Abnormal Psychol 1991;100:391-398.

[4] Hamby, Sherry. On defining violence, and why it matters. US: Educational Publishing Foundation. Retrieved from (Apr 2017) https://doi.org/10.1037/vio0000117

[5] Bandura, A. National Inst of Mental Health. Social foundations of thought and action: A social cognitive theory.(1986). 
[6] CATHY SPATZ WIDOM. VARIETIES OF VIOLENT BEHAVIOR. CRIMINOLOGY. Retrieved from https://onlinelibrary.wiley.com/doi/full/10.1111/17 45-9125.12046

[7] R.J.R. Blair,K.S. Peschardt,S. Budhani,D.G.V. Mitchell,D.S. Pine. The development of psychopathy. The Journal of Child Psuchology and Psychiatry. Retrieved from (22 February 2006). https://doi.org/10.1111/j.1469-7610.2006.01596.x

[8] Bandura, A. National Inst of Mental Health. Social foundations of thought and action: A social cognitive theory.(1986). 